\section{Análisis crítico de un artículo: antibióticos en otitis media aguda, ¿son necesarios?, ¿existe algún subgrupo que se beneficia?}

\author{
PAMELA ROJAS ${ }^{1}$, GABRIEL RADA ${ }^{2,3,4}$
}

\section{Critically appraised article}

Sanders S, Glasziou PP, Del Mar CB, Rovers MM.
Antibiotics for acute otitis media in children. Cochrane Database
of Systematic Reviews 2004, Issue 1. Art. No.: CD000219.
(Assessed as up-to-date: 8 Nov 2008)

Background: Acute otitis media (AOM) is one of the most common diseases in early infancy and childhood. Antibiotic use for AOM varies from $56 \%$ in the Netherlands to 95\% in the USA and Australia. Objectives: To assess the effects of antibiotics for children with AOM. Search strategy: We searched the Cochrane Central Register of Controlled Trials (CENTRAL) (The Cochrane Library, 2008, issue 2) which contains the Acute Respiratory Infections (ARI) Group's Specialized Register; MEDLINE (1966 to June week 4 2008); OLDMEDLINE (1958 to 1965); EMBASE (January 1990 to July 2008); and Current Contents (1966 to July 2008). Selection criteria: Randomised controlled trials comparing 1) antimicrobial drugs with placebo 2) immediate antibiotic treatment with observational treatment approaches in children with AOM. Data collection and analysis: Three review authors independently assessed trial quality and extracted data. Main results: We found 10 trials (2928 children) from high income countries with low risk of bias. Pain was not reduced by antibiotics at 24 hours, but was at two to seven days, (relative risk (RR) $0.72 ; 95 \%$ confidence interval 0.62 to 0.83). However four trials (1271 children) comparing antibiotics prescribed immediately rather than initial observation found no difference at three to seven days. Antibiotics did not reduce tympanometry, perforation or recurrence. The only case of mastoiditis was in an antibiotic treated child. Vomiting, diarrhoea or rash was higher in children taking antibiotics (RR 1.37; 95\% CI 1.09 to 1.76). Individual patient data meta-analysis of a subset of the included trials found antibiotics to be most beneficial in children: aged less than two; with bilateral AOM and with both AOM and otorrhoea. Authors' conclusions: Antibiotics slightly reduce the number of children with acute middle ear infection experiencing pain after a few days. However, most (78\%) settle spontaneously in this time, meaning 16 children must be treated to prevent one suffering ear pain. This benefit must be weighed against the possible harms: 1 in 24 children experience symptoms caused by antibiotics. Antibiotics are most useful in children under two years of age, with bilateral AOM, and with both $A O M$ and discharging ears. For most other children with mild disease, an expectant observational approach seems justified. We have no data on populations with higher risks of complications.

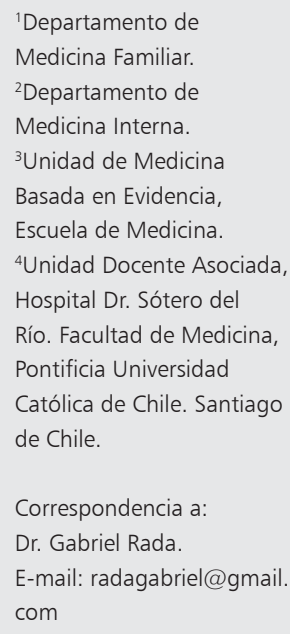




\section{Conclusión de los revisores}

Los beneficios de los antibióticos en pacientes con otitis media aguda no justifican su prescripción en la mayoría de los pacientes. En pacientes con otorrea, y en menores de 2 años con compromiso bilateral, parece justificada su utilización.

\section{Pregunta clínica}

¿Cuáles son los beneficios y efectos adversos del uso de antibióticos en niños con otitis media aguda?

\section{Introducción}

La otitis media aguda (OMA) corresponde a la infección viral o bacteriana del oído medio ${ }^{1}$. En algunos países es la principal causa por la que se indican antibióticos en población infantili2; lo que genera un consumo importante de recursos y contribuye al proceso de desarrollo de resistencia bacteriana.

La indicación de antibióticos en el tratamiento de las OMA infantiles aún genera controversia. Pese a que el grueso de la evidencia es clara en relación a no utilizarlos en todos los pacientes, y reservarlos sólo para un grupo que parece beneficiarse de esta práctica, muchos clínicos continúan haciéndolo.

Este análisis crítico revisará esta controversia, con particular interés en el análisis de subgrupos que podrían beneficiarse del tratamiento antibiótico. El artículo precedente sirve de sustento teórico a lo aquí expuesto 3 .

\section{Características del estudio}

Tipo de estudio: Revisión sistemática de estudios clínicos randomizados. La búsqueda inicial se realizó en 4 bases de datos electrónicas, y fue actualizada en julio de 2008, incluyendo CENTRAL, MEDLINE y EMBASE. Adicionalmente, se realizó una búsqueda en las referencias de los estudios encontrados. No se limitó la búsqueda por idioma ni por el estado de publicación.

Se incluyeron estudios que evaluaran niños con otitis media, entre 1 mes y 15 años, en los que se utilizara cualquier antibiótico, comparado con placebo o con conducta expectante.

La revisión identificó 11 estudios, cuyos participantes tenían entre 2 meses y 15 años. El porcentaje de hombres y mujeres fue similar. Se realizó un análisis de pacientes individuales (datos disponibles para 6 estudios) para evaluar el efecto en el subgrupo de niños menores de 2 años, en aquellos que presentaban otorrea, y en los con compromiso bilateral.

\section{Validez (riesgo de sesgo) del estudio}

\begin{tabular}{ll|}
\hline 1. Pregunta específica y focalizada & SI \\
\hline 2. Búsqueda amplia y completa & Parcialmente \\
3. Criterios de inclusión explícitos y adecuados & SI \\
4. Evaluación de calidad de los estudios incluidos & SI \\
5. Reproducibilidad del proceso & SI \\
6. Evaluación de heterogeneidad & SI \\
* No se buscó en resúmenes de congresos, en registros de compañías farmacéuticas ni se \\
contactó a expertos.
\end{tabular}


Antibióticos en otitis media aguda - P. Rojas et al

Resultados

\begin{tabular}{|c|c|c|c|c|c|c|}
\hline \multirow{3}{*}{ Outcome } & \multicolumn{6}{|c|}{ Antibióticos para la otitis media en niños } \\
\hline & \multicolumn{3}{|c|}{ Efectos absolutos } & \multirow{2}{*}{$\begin{array}{l}\text { Efecto } \\
\text { relativo } \\
\text { (IC 95\%) }\end{array}$} & \multirow{2}{*}{$\begin{array}{c}\text { n de } \\
\text { pacientes } \\
\text { (estudios) }\end{array}$} & \multirow{2}{*}{$\begin{array}{l}\text { Comenta- } \\
\text { rios }\end{array}$} \\
\hline & $\begin{array}{c}\text { Eventos SIN } \\
\text { antibióticos } \\
(\%)\end{array}$ & $\begin{array}{c}\text { Eventos CON } \\
\text { antibióticos } \\
(\%)\end{array}$ & Diferencia & & & \\
\hline $\begin{array}{l}\text { Dolor entre los } \\
\text { días } 2 \text { y } 7\end{array}$ & 257 por 1.000 & $\begin{array}{c}185 \text { por } 1.000 \\
\text { (159 to } 213)\end{array}$ & $\begin{array}{l}72 \text { pacientes } \\
\text { de cada } 1.000 \\
\text { estarían sin dolor } \\
\text { entre los días } 2 \\
\text { y } 7 \text { gracias a los } \\
\text { antibióticos }\end{array}$ & $\begin{array}{c}\text { RR } 0,72 \\
(0,62 \text { a } 0,83)\end{array}$ & $\begin{array}{c}2.791 \\
(10)\end{array}$ & $\begin{array}{l}\text { Existe eviden- } \\
\text { cia de alta } \\
\text { calidad para } \\
\text { este outcome }\end{array}$ \\
\hline $\begin{array}{l}\text { Dolor, fiebre } \\
\text { o ambos en } \\
\text { menores de } 2 \\
\text { años con OMA } \\
\text { bilateral }\end{array}$ & 552 por 1.000 & $\begin{array}{c}304 \text { por } 1.000 \\
(226 \text { a } 403)\end{array}$ & $\begin{array}{l}248 \text { pacientes } \\
\text { de cada } 1.000 \\
\text { estarían sin dolor } \\
\text { entre los días } 2 \\
\text { y } 7 \text { gracias a los } \\
\text { antibióticos }\end{array}$ & $\begin{array}{c}\operatorname{RR} 0,55 \\
(0,41 \text { a } 0,73)\end{array}$ & $\begin{array}{l}1.643 \\
(6)\end{array}$ & $\begin{array}{l}\text { Análisis de } \\
\text { subgrupo }\end{array}$ \\
\hline $\begin{array}{l}\text { Dolor, fiebre } \\
\text { o ambos en } \\
\text { pacientes con } \\
\text { otorrea }\end{array}$ & 600 por 1.000 & $\begin{array}{c}234 \text { por } 1.000 \\
(138 \text { a } 402)\end{array}$ & $\begin{array}{l}296 \text { pacientes } \\
\text { de cada } 1.000 \\
\text { estarían sin dolor } \\
\text { entre los días } 2 \\
\text { y } 7 \text { gracias a los } \\
\text { antibióticos }\end{array}$ & $\begin{array}{c}\text { RR } 0,39 \\
(0,23 \text { a } 0,67)\end{array}$ & $\begin{array}{c}1.643 \\
(6)\end{array}$ & $\begin{array}{l}\text { Análisis de } \\
\text { subgrupo }\end{array}$ \\
\hline $\begin{array}{l}\text { Efectos adversos: } \\
\text { vómitos, diarrea } \\
\text { o rash }\end{array}$ & 113 por 1.000 & $\begin{array}{c}156 \text { por } 1.000 \\
(123 \text { a } 199)\end{array}$ & $\begin{array}{l}43 \text { pacientes de } \\
\text { cada } 1.000 \text { trata- } \\
\text { dos presentarían } \\
\text { efectos adversos } \\
\text { por los antibióticos }\end{array}$ & $\begin{array}{c}\text { RR 1,38 } \\
(1,09 \text { a } 1,76)\end{array}$ & $\begin{array}{l}1.401 \\
(5)\end{array}$ & $\begin{array}{l}\text { Existe } \\
\text { evidencia de } \\
\text { moderada } \\
\text { calidad para } \\
\text { este outcome }\end{array}$ \\
\hline
\end{tabular}

$\mathrm{IC}=$ Intervalo de confianza, $\mathrm{RR}=$ Riesgo relativo.

\section{Comentarios}

\section{Comentarios acerca de la validez (riesgo de sesgo)}

Es una revisión sistemática de buena calidad, que cumple con los estándares de la Colaboración Cochrane. La búsqueda es reciente, sin embargo, podría haber sido más exhaustiva en la búsqueda de estudios no publicados. Afortunadamente, al comparar con otras revisiones sistemáticas en esta área, parece que no han quedado fuera estudios relevantes ${ }^{4-6}$.

Es interesante destacar que se realizó un análisis de subgrupo utilizando los datos de pacientes individuales. Lamentablemente, como suele ocurrir cuando se intenta utilizar este método, no todos los autores de los estudios entregaron los datos crudos (raw data) para poder realizarlo. Existen múltiples iniciativas proponiendo que este tipo de datos se hagan públicos. Destacamos la reciente declaración de la Colaboración Cochrane, abogando por un mayor acceso a estos datos ${ }^{7}$.

\section{Comentarios acerca de los resultados:}

Los autores hacen una opción clara por aquellos estudios centrados en medir outcomes importantes para el paciente, lo cual facilita la ponderación de 
beneficios y riesgos. En la mayoría de los desenlaces medidos, el tratamiento antibiótico comparado con placebo, o con una conducta expectante, no ofrece beneficios significativos. De cada 1.000 pacientes que tratemos con una OMA, 72 estarán sin dolor o fiebre entre el $2^{\circ}$ y $7^{\circ}$ día gracias a los antibióticos y 43 presentarán un efecto adverso (vómitos, náuseas o diarrea). En la gran mayoría de los niños los síntomas se resolverán sin importar el uso o no uso de antibióticos. De hecho, un tercio de los pacientes resuelve sus síntomas en las primeras 24 horas.

Es interesante destacar el esfuerzo realizado en esta revisión, por analizar el efecto sobre algunos subgrupos que pueden beneficiarse ${ }^{8}$. Una revisión de nuestro grupo analiza los aspectos a considerar al momento de leer un análisis de subgrupo ${ }^{3}$. Aplicaremos esos conceptos en esta revisión sistemática.

El análisis realizado propone la hipótesis que ciertos subgrupos podrían beneficiarse más que otros del uso de antibióticos. Esto, en base a lo reportado ampliamente en la literatura y a una herramienta de predicción con múltiples variables.

El primer punto a considerar, al plantear la existencia de un efecto de subgrupo, es determinar si los riesgos relativos (RR) difieren entre sí (no los absolutos). En este caso, el RR de los 2 subgrupos presentados en la Tabla es claramente diferente, por lo que es razonable plantear un efecto de subgrupo. La pregunta que sigue entonces es; ¿podemos confiar en que este efecto de subgrupo es real? (y no sea producto del azar, o de la existencia de error sistemático o sesgo). Una serie de aspectos del diseño del estudio nos permiten hacer este juicio:

- En el protocolo de la revisión (a priori) está claramente especificado cuáles son los subgrupos a evaluar y cuál es la dirección del efecto esperado. Generalmente las revisiones sistemáticas dependen de que esto haya sido establecido en todos los estudios individuales. Sin embargo, al utilizar la técnica de metaanálisis de paciente individual se transforman los diferentes estudios en un solo gran estudio, en el que se puede analizar apropiadamente este efecto. Esta técnica también permite soslayar otra dificultad inherente a las revisiones sistemáticas, que es que las comparaciones entre estudios (interestudio) son menos confiables que aquellas hechas dentro de un mismo estudio (intraestudio).

- El número de subgrupos explorados es bajo (edad, compromiso bilateral y presencia de otorrea). De hecho, es excepcional que los autores se centren en un número tan acotado de hipótesis.

En segundo lugar debemos fijarnos en el modo de analizar los datos:

- El mismo grupo de autores reporta en otra publicación el resultado detallado del metanálisis de paciente individual ya mencionado (8). Ahí se describe claramente, mediante un test de interacción, que la diferencia entre algunos subgrupos es significativa.

- Por otro lado, mediante un análisis de regresión múltiple, se comprueba que estas diferencias entre subgrupos son independientes de otros factores.

Finalmente, para confiar en un efecto de subgrupos se deben tener presentes aspectos relacionados con el contexto general:

- La magnitud del efecto observada es considerable. Las diferencias en términos absolutos son notorias, pero lo más importante es que las diferen- 
cias expresadas en una medida de riesgo relativo (RR) sean importantes también.

- Finalmente, un aspecto necesario (pero no suficiente) para confiar en un efecto de subgrupo es que haya plausibilidad biológica. En este caso, existe demostración clara que la otorrea es más frecuente en las otitis de origen bacteriano, así como también es más frecuente el aislamiento de bacterias en las muestras timpánicas de menores de 2 años, y en pacientes con compromiso bilateral ${ }^{10}$.

\section{Comentarios acerca de la aplicabilidad}

Los outcomes elegidos consideran una amplia gama de resultados, clínicamente relevantes. Se incluyen resultados relevantes por su potencial gravedad para el paciente (complicaciones, efectos adversos del uso de antibióticos); resultados menos graves en términos de su trascendencia clínica, pero de importancia para el paciente y su familia (duración del dolor y fiebre); y resultados a largo plazo (recurrencias). No quedan outcomes clínicamente relevantes fuera del análisis.

Los hallazgos de esta revisión son aplicables a la población general que se atiende en servicios de salud públicos y privados de nuestro país. Los resultados deben ser aplicados prudentemente en aquellos pacientes en los cuales no es posible asegurar el acceso expedito a servicios de salud (sectores rurales más aislados, dificultades serias para el traslado, etc); o aquellos niños cuyos cuidadores presenten dificultades importantes para reconocer signos de alarma (ej. retraso mental; deprivación cultural grave). El estudio no incluye a pacientes con alto riesgo de presentar complicaciones, de modo que los resultados no son aplicables a ellos (ej. niños inmunosuprimidos). También se debe tener precaución en poblaciones con mayor riesgo de mastoiditis (por ej. algunos países de bajos ingresos). En ninguno de los estudios encontrados por la revisión, todos ellos realizados en países de altos ingresos, se presentó esta complicación.

Es interesante la discusión con respecto a qué decisión tomar cuando existen beneficios y riesgos de magnitud similar, tal como ocurre en la mayor parte de los niños con OMA. Si bien podría decirse que es razonable una recomendación general en contra del uso de antibióticos, es importante considerar aspectos relacionados con la preferencia de los padres, el umbral de dolor de los niños, su respuesta a analgesia o antiinflamatorios habituales, etc.

La situación en nuestro país parece estar dividida, entre padres que prefieren tratar con antibióticos a sus niños y los que prefieren evitarlos en la medida de lo posible. Aun en este grupo, sin embargo, el uso de antibióticos en niños con OMA es ampliamente aceptado. Por otra parte, muchos clínicos manifiestan a priori su temor con respecto a que los padres rechacen la alternativa de un tratamiento sin antibióticos. Esto, y en algunos casos el temor a potenciales acciones judiciales si el niño se complica, motivan su prescripción. Este problema no es exclusivo del medio chileno, y ha sido ampliamente reconocido y abordado en otros países. El solo conocimiento de la evidencia no es en ningún caso suficiente para contrarrestar una costumbre tan arraigada, y existen diversas intervenciones relacionadas con los sistemas de provisión de salud que deben ser consideradas por quienes se interesen por abordar esta problemática $^{11}$. 


\section{Referencias}

1. Ramakrishnan K, Sparks R, Berryhill W. Diagnosis and treatment of otitis media. Am Fam Physician 2007; 76 (11): 1650-8.

2. Gould J, Matz P. Otitis Media. Pediatrics in Review 2010; 3: 102-16.

3. Candia R, Rivera S, Neumann I. Análisis de subgrupos: ¿Mejoran la interpretación de los resultados o nos inducen al error? Rev Med Chile 2012; 140: 685-92.

4. Koopman L, Hoes AW, Glasziou PP, Appelman CL, Burke P, McCormick DP, et al. Antibiotic therapy to prevent the development of asymptomatic middle ear effusion in children with acute otitis media: a metaanalysis of individual patient data. Arch Otolaryngol Head Neck Surg 2008; 134 (2): 128-32.

5. Vouloumanou EK, Karageorgopoulos DE, Kazantzi MS, Kapaskelis AM, Falagas ME. Antibiotics versus placebo or watchful waiting for acute otitis media: a metaanalysis of randomized controlled trials. J Antimicrob Chemother 2009; 64 (1): 16-24.
6. Coker TR, Chan LS, Newberry SJ, Limbos MA, Suttorp MJ, Shekelle PG, et al. Diagnosis, microbial epidemiology, and antibiotic treatment of acute otitis media in children: a systematic review. JAMA 2010; 304 (19): 2161-9.

7. Gøtzsche PC, Tovey D. We need access to all data from all clinical trials. Cochrane Database Syst Rev 2011; 12: ED000035.

8. Rovers MM, Glasziou P, Appelman CL, Burke P, McCormick DP, Damoiseaux RA, et al. Antibiotics for acute otitis media: a meta-analysis with individual patient data. Lancet 2006; 368 (9545): 1429-35.

9. Sun $\mathrm{X}$, Briel M, Walter SD, Guyatt GH. Is a subgroup effect believable? Updating criteria to evaluate the credibility of subgroup analyses. BMJ 2010; 340: c117.

10. Palmu AA, Herva E, Savolainen H, Karma P, Mäkelä PH, Kilpi TM. Association of clinical signs and symptoms with bacterial findings in acute otitis media. Clin Infect Dis 2004; 38: 234-42.

11. Arnold SR, Straus SE. Interventions to improve antibiotic prescribing practices in ambulatory care. Cochrane Database Syst Rev 2005; (4): CD003539. 